\title{
TERRITÓRIOS VIVIDOS NO ESPAÇO URBANO DE NATAL-RN: AS CONDIÇÕES EXISTENCIAIS NO CONTEXTO DA PANDEMIA DA COVID-19
}

\author{
LIVED TERRITORIES IN THE URBAN AREA OF NATAL: EXISTENTIAL CONDITIONS IN \\ THE COVID-19 PANDEMIC CONTEXT
}

Hugo Arruda de MORAIS ${ }^{1}$, Ana Clara Celestino BELCHIOR ${ }^{2}$, Yan de Oliveira MACHADO ${ }^{3}$

\begin{abstract}
Palavras-chave:
Pandemia;

Território vivido;

Espaço urbano;

Natal/RN.

RESUMO

A expansão do novo coronavírus (COVID-19) é mais uma das etapas do acontecer histórico da sociedade global e, como todo fenômeno, ganhou dimensões espaciais. No caso brasileiro, a forte expansão das infecções entre os cidadãos se dá intensamente nos espaços urbanos, porém, afetando de maneira muito desigual. Nesse sentido, o objetivo deste ensaio consiste em compreender a condição existência dos territórios vividos em Natal, no atual contexto da pandemia da COVID-19. Em termos de procedimentos metodológicos, foram sistematizados e espacializados indicadores socioeconômicos dos bairros da capital potiguar, numa busca de caracterizar os territórios vividos. Os resultados indicam um cenário em que as condições existenciais tendem a um agravamento com 0 aumento no número de casos e mortes por conta do novo coronavírus.
\end{abstract}
Keywords:
Pandemic;
Territory lived;
Urban space;
Natal/RN.

\section{ABSTRACT}

The expansion of the new coronavirus (COVID-19) is one more historical fact in the global society and, like every phenomenon, has gained spatial dimensions. In Brazil, the spread of infections among citizens occurs intensely in urban spaces. However, affecting unequally each local. Consequently, the objective of this essay is to understand how the current context of the COVID-19 pandemic affects the condition of the lived territories in Natal. As a methodological procedure, we systematized and spacialized the socioeconomic indicators of neighborhoods in the capital city. The results indicate that the increase of cases and deaths by coronavirus tend to worsen the living conditions of the population.

\footnotetext{
1Professor Adjunto do Departamento de Geografia da Universidade Federal do Rio Grande do Norte (UFRN), Campus Natal. E-mail: hugo.geografia@yahoo.com.br

${ }^{2}$ Graduanda em Geografia pela Universidade Federal do Rio Grande do Norte (UFRN). E-mail: clarae260@gmail.com

${ }^{3}$ Graduando em Geografia pela Universidade Federal do Rio Grande do Norte (UFRN). E-mail: yanmachado21@gmail.com
} 


\section{INTRODUÇÃO}

De acordo com a Organização Mundial da Saúde (OMS), o surto da síndrome respiratória aguda grave SARS-CoV-2 (COVID-19) constitui uma emergência de saúde pública de importância internacional. 0 novo coronavírus abrange um grupo de vírus que causa problemas respiratórios sérios, podendo evoluir para casos mais graves, especialmente em idosos e pessoas com comorbidades, apresentando, inclusive, grande capacidade de propagação proporcionada pelo contato entre pessoas aglomeradas, bem como pelo contato entre pessoas e superfícies ou objetos contaminados (World Health Organization, 2020).

No mundo, os primeiros registros de casos da COVID-19 ocorreram desde o final de 2019 a partir da província de Hubei na China, especificamente da cidade de Wuhan, de onde, a partir do início de 2020, expandiu-se pelo mundo constituindo-se como mais uma das grandes pandemias. No caso do Brasil, os primeiros registros se deram nos primeiros meses do ano de 2020, a partir de "fluxos aéreos internacionais" (Pereira, Diniz, Rocha, \& Oliveira Júnior, 2020, p. 434), fazendo com que a maioria da população tivesse que adotar medidas como o distanciamento social, além de ações de conscientização, mudança e inserção de novos hábitos de higienização, uma vez que a rápida propagação de infectados e de mortes pressionou os sistemas de saúde público e privado em todo território nacional.

Como todo fenômeno, a COVID-19 ganhou dimensões espaciais, fazendo com que a curva epidemiológica do número de casos e de óbitos seguisse um ritmo muito rápido e de forma diferente em várias regiões do país, mostrando que toda a dinâmica de transmissão segue diferentes escalas (Candido et al., 2020), principalmente, nos espaços urbanos.

Nesse caminho, as condições sociais, culturais, políticas, econômicas, ambientais, etc., inerentes ao uso dos diversos territórios influem, dialeticamente, na saúde da população. Por isso, as situações existenciais dos espaços urbanos possibilitam explicar muitos fatores de disseminação e agravamento dos casos e mortes ligadas à COVID-19.

Em sendo assim, se olharmos para a cidade de Natal-RN, até a divulgação, pela Secretaria Municipal de Saúde, a partir do Departamento de Vigilância em Saúde, do Boletim Epidemiológico No 13, em 13 de julho de 2020, a capital potiguar contava com 15.316 casos confirmados e 562 óbitos pela COVID-19. Porém, a maioria dos focos de disseminação da doença e de vítimas fatais se concentrando em áreas como as zonas Norte e Oeste da cidade, regiões com sérios problemas de natureza social e territorial.

Diante desse contexto de rápida expansão espacial do vírus nos diversos territórios dos espaços urbano e, em especial, na capital potiguar, cabe questionar: Quais as condições existências dos territórios vividos em Natal-RN, no atual contexto de pandemia da COVID-19? As condições existências possibilitam um cenário propicio para a maior incidência de casos de mortes e óbitos pela COVID-19? 
Nesse sentido, o objetivo desse ensaio consiste, principalmente, em compreender a condição existência dos territórios vividos em Natal, no atual contexto da pandemia da COVID-19. Partimos da perspectiva de que a espacialização dos casos de infecção e mortes pelo novo coronavírus na capital potiguar agrava a condição desigual e injusta vivida cotidianamente.

A realização deste trabalho fundamentou-se, basicamente, numa abordagem metodológica calcada na análise espacial da cidade do tema delimitado. Ao buscarmos fazer uma "leitura geográfica sobre a cidade" (Carlos, 2017), centramos nossas reflexões no processo de construção humana da cidade, tendo nos territórios vividos a relação existencial da sociedade com a natureza, principalmente, as classes mais subalternas. Nesse sentido, e diante de um contexto de pandemia da COVID-19, os territórios vividos se apresentam como as áreas onde vivem as classes mais subalternas, constituídos por trabalhadores mais pobres da sociedade, e que sofrem com a carência de bens e serviços que condicionam uma existência cotidiana difícil (Morais \& Castilho, 2020).

O presente texto é fruto das reflexões iniciais e exploratórias acerca da pandemia da COVID-19 na realidade de Natal-RN e que ainda se apresenta como uma questão atual e com inúmeras incertezas quanto ao seu futuro. Logo, as assertivas são colocadas dentro de um processo onde os fatos se encontram em curso. Destaca-se, ainda, que esta pesquisa é produto de uma das etapas da pesquisa de extensão, intitulada: A concretização do espaço do cidadão em Natal-RN: uma reflexão no contexto da pandemia da COVID-19. O referido projeto está sendo realizado numa parceria entre o Departamento de Geografia (DGEUFRN) com o grupo de pesquisa Movimentos Sociais e Espaço Urbano (MSEU), do Departamento de Geografia da UFPE.

\section{MÉTODO}

Numa perspectiva analítica de enxergar as condições desiguais e injustas vivenciadas pelas classes mais subalternas, no contexto da COVID-19, a primeira etapa da pesquisa foi identificar os territórios vividos, ou seja, aqueles territórios mais vulneráveis socialmente e, por consequência, mais propícios a uma rápida expansão de casos e óbitos causados pela doença.

Para tal, foram consultados os sites do Plano Diretor de Natal (https://natal.rn.gov.br/semurb/planodiretor/), em que no documento "Conheça Melhor Seu Bairro" há informações sobre o número de habitantes por bairros, a renda per capita ( $n^{0}$ de salários) e taxa de alfabetização, e no Instituto Brasileiro de Geografia e Estatística (IBGE) para coletar dados referente aos aglomerados subnormais de Natal para o ano de 2019. Munidos dessas informações, indentificamos as áreas 
e as características socioeconômicas dos territórios vividos na capital potiguar, o que nos permitiu observar as desigualdades existentes e compartilhadas cotidianamente pela classe trabalhadora.

É importante destacar que essa pesquisa está em andamento e que as próximas etapas se darão seguindo os passos descritos. Primeiro, a partir do levantamento dos dados epidemiológicos por bairro na cidade de Natal-RN. Esses dados serão obtidos no sítio eletrônico da Prefeitura Municipal de Natal (https://coronavirus.natal.rn.gov.br/), especificamente na seção que apresenta o Boletim epidemiológico na Secretaria Municipal de Saúde, a partir do Departamento de Vigilância em Saúde, dentre o período de 15 de abril até 13 de julho de 2020.

A partir do levantamento de dados epidemiológicos sobre a COVID-19 no município, poderemos identificar as áreas com maior e menor concentração de casos e óbitos. Como também, identificar se essas áreas se confundem exatamente com os territórios das populações mais pobres de Natal. Com isso, construiremos mapas temáticos que mostrem a espacialização do novo coronavírus em Natal, o que nos permitiu fazer uma análise da espacialização da doença, correlacionando esses indicadores de casos e óbitos por COVID-19 e características socioeconômicas da população.

\section{RESULTADOS E DISCUSSÃO}

A partir dos primeiros dados levantados, verificou-se que o município de Natal conta com uma população estimada em 2017 de 885.173 habitantes, distribuídos em 167,401 Km² de unidade territorial da capital. 0 referido município é constituído de 36 bairros distribuídos em quatro regiões administrativas (Norte, Sul, Leste e Oeste) (Figura 1), onde cada uma dessas áreas apresenta diferentes características territoriais em termos demográficos, econômicos e de infraestrutura. 


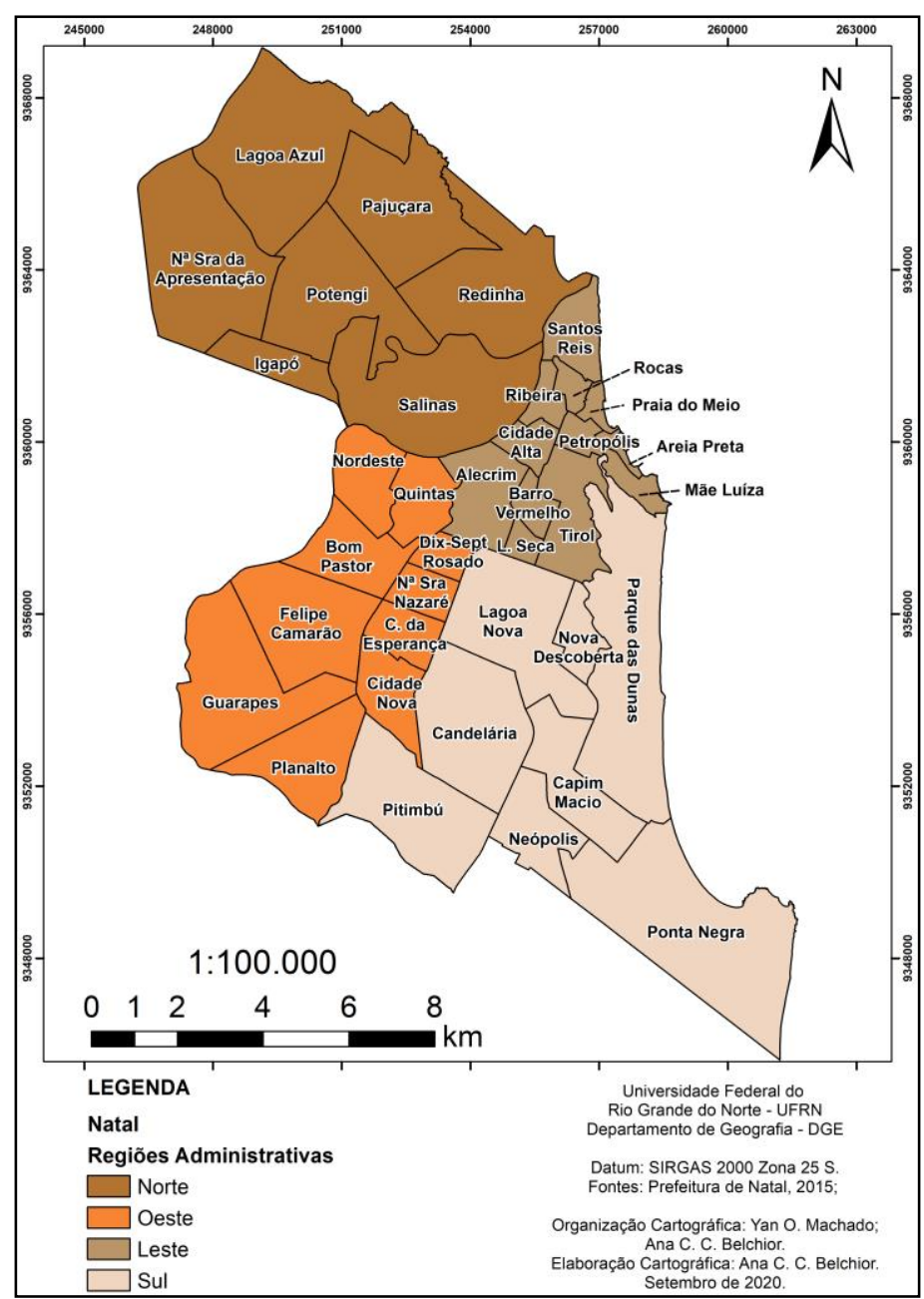

Figura 1 - A espacialização da cidade do Natal-RN - 2020.

Fonte: Prefeitura do Natal (2015).

Partindo da perspectiva que os territórios vividos são mais afetados, principalmente, pelo número de casos de pessoas contaminadas e óbitos pelo novo coronavírus, agravando a condição existencial, principalmente, por serem áreas de população e com grandes focos de pobreza e precária inclusão, identificamos os territórios onde se encontram a população com as menores médias salariais e de alfabetização e as áreas em que se encontram os maiores aglomerados subnormais da cidade do Natal (Figura 2). 


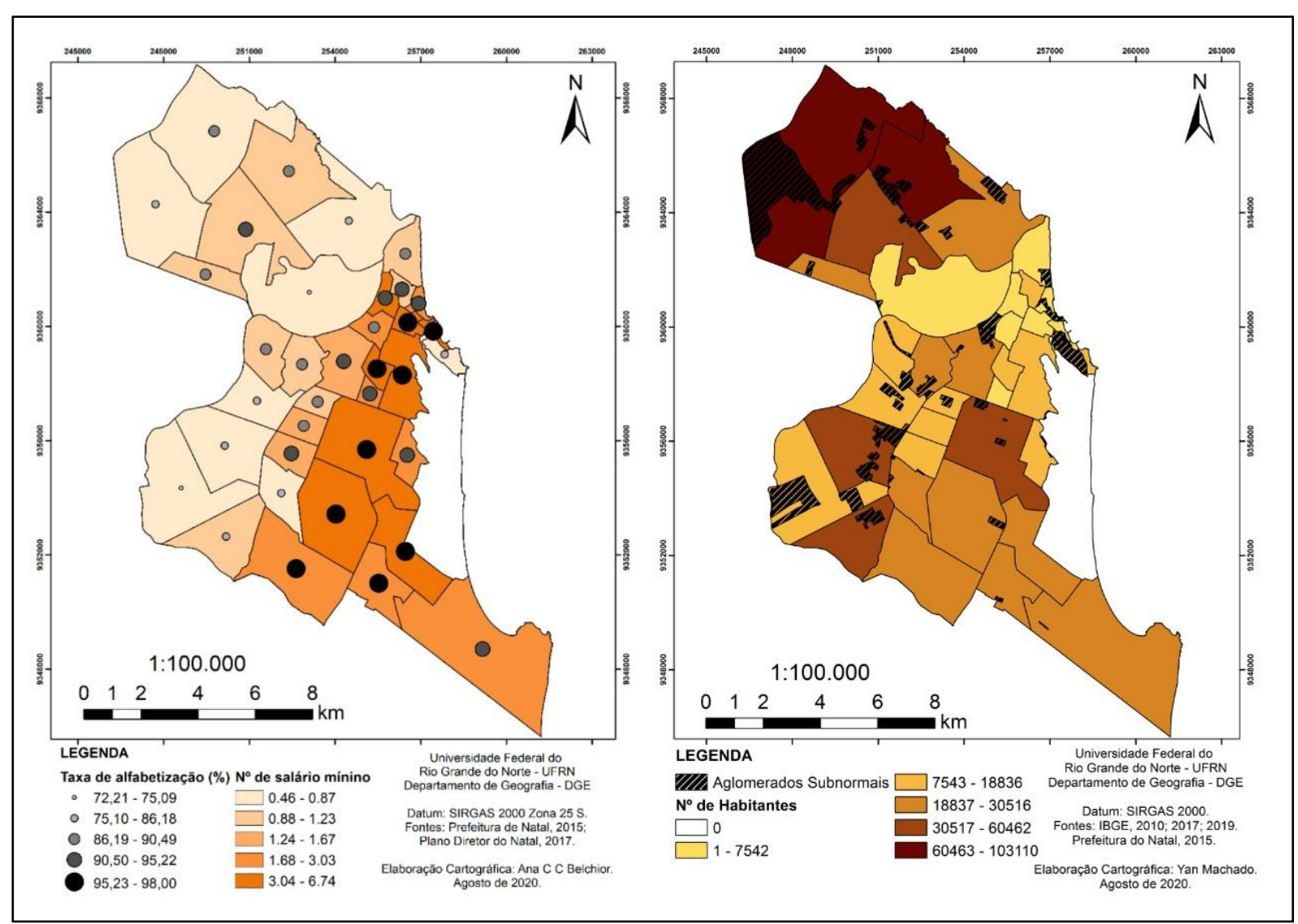

Figura 2 - Distribuição espacial da renda e alfabetização das áreas de aglomerados subnormais e o número de habitantes por bairro - Natal-RN - 2020.

Fonte: IBGE (2010; 2017; 2019), Prefeitura do Natal $(2015 ; 2017)$.

Os dados mostram que o município de Natal conta com aglomerados subnormais distribuídos em todas as zonas político-administrativas. São ao todo 33.372 domicílios nessa condição, onde $36 \%$ encontramse na Zona Oeste, 27\% na Zona Norte, 23\% na Zona Leste e apenas 14\% na Zona Sul. Contudo, vale destacar que, apesar da Zona Oeste apresentar a maior quantidade de aglomerados, é na Zona Norte que há o maior número de domicílios, apresentando $48 \%$ dos domicílios, enquanto a Zona Oeste possui $27 \%$. Se considerarmos a média populacional de 2,9 pessoas por domicílios para Natal, segundo a Pesquisa Nacional por Amostras de Domicílios, estariam nessas áreas aproximadamente 96.778 pessoas.

Ao mesmo tempo, é possível identificar que em relação à renda, as zonas Norte e Oeste são as que possuem as menores médias em números de salários mínimos, 0.92 e 0,99, respectivamente. São valores inferiores a 1 salário e ainda inferiores à média da cidade do Natal (1,78, segundo o Plano Diretor). Sendo ainda, as Zonas Norte e Oeste, as regiões onde se encontram os bairros com as menores médias salariais, sendo o bairro Salinas na Zona Norte com 0,46 e o bairro Guarapes na Zona Oeste com 0,53 salários mínimos. As Zonas Sul e Leste possuem valores superiores ao da cidade. A região Leste possui uma média 
de 2,86, e a Zona Sul, 3,45. Mesmo não sendo a Zona Leste a região administrativa com a maior média salarial, é nela onde se localizam os bairros com os melhores números: Tirol $(6,46)$ e Petrópolis $(6,74)$.

Também são as Zonas Norte e Oeste que detêm as menores médias de alfabetização $(88 \%$ e $85,83 \%$, respectivamente). Os mesmos bairros que possuem as menores médias salariais da cidade se repetem e são as menores médias de alfabetização. Salinas na Zona Norte possui 75,09\%, e Guarapes, $72,21 \%$ na Zona Oeste. Dessa forma, as Zonas Leste e Sul apresentam as maiores médias de alfabetização. A região Sul tem as melhores médias da cidade com $96,24 \%$ de sua população alfabetizada, já a região Leste 93,07\%. O bairro com a média mais próxima de 100\% é Capim Macio com 97,98\%, localizado na Zona Sul.

Tais dados deixam claro que as Zonas Norte e Oeste da capital potiguar são as áreas que se caracterizam como territórios vividos. Percebe-se pelos dados apresentados que as duas regiões administrativas apresentam uma natureza social e territorial que possibilita uma propagação espacial da COVID-19, uma vez que as especificidades sociais e econômicas inerentes ao uso dos territórios influem, dialeticamente, na saúde humana. Nesse sentido, concordamos com Chasles (2019) quando este identifica que além do comportamento individual das pessoas, o ordenamento territorial tem um peso muito grande na saúde humana.

\section{CONCLUSÕES}

Se tratando de uma pesquisa de carácter exploratório e com as inúmeras incertezas que circundam a pandemia da COVID-19 dentro da realidade brasileira e da capital potiguar, as considerações colocadas aqui estão dentro de um movimento e que pode passar por mudanças constantes, fruto da dinâmica do acontecer da sociedade.

Dessa forma, alguns dos resultados já mostram uma tendência de que as áreas da cidade do Natal com menores médias salariais e com os menores índices de alfabetização são as mesmas em que se encontram os maiores aglomerados subnormais na capital potiguar. São as zonas Norte e Oeste as que se apresentam como os territórios vividos no espaço urbano de Natal-RN, uma vez que as características sociais e espaciais possibilitam que esses territórios se configurem como espaços constituídos por trabalhadores mais pobres da cidade, ou seja, "lugares da escassez, das ausências, das carências" (Morais \& Castilho, 2020, p. 39).

Os novos caminhos de análise da pesquisa se darão no sentido de espacializar os casos e óbitos, no período que se estende do primeiro caso registrado na capital potiguar (12/03/2020) até o dia 13 de julho. As análises buscaram fazer uma correlação entre a incidência da COVID-19 em Natal-RN e o agravamento das 
condições existentes nos territórios vividos. As primeiras análises já apontam para um cenário onde se observa que as zonas com os maiores déficits em relação aos valores apresentados de renda, alfabetização e abastecimento de água apresentam a disseminação e mortalidade por conta da doença, tendendo a agravar a condição desigual e injusta vivida cotidianamente.

\section{REFERÊNCIAS}

Candido, D. D. S., Watts, A., Abade, L., Kraemer, M. U., Pybus, O. G., Croda, J., ... \& Faria, N. R. (2020). Routes for COVID-19 importation in Brazil. Journal of Travel Medicine, 27(3), taaa042.

Carlos, A. F. A. (2020). Espaço Urbano. São Paulo: Labur Edições, 2007, 184 p.

Chasles, V. (2019). Introduction générale: territoire et santé, des liens pluriels. Santé et territoire, une réflexion géographique. Lyon: Université Jean Moulin Lyon 3/Faculté des Lettres et Civilisations.

IBGE, Instituto Brasileiro de Geografia e Estatística. (2019). Aglomerados Subnormais. Disponível: https://www.ibge.gov.br/geociencias/organizacao-doterritorio/15788-aglomerados-

subnormais.html?edicao=27720\&t=sobre.

Morais, H. A. de, \& de Castilho, C. J. M. (2020). DESENVOLVIMENTO - UMA QUESTÃO AINDA PERTINENTE?. Sociedade e Território, 32(1), 152-173.

Pereira, V. H. C., Diniz, M. T. M., Rocha, G. C., \& Oliveira Júnior, M. A. C. D. (2020). Identificação das rotas iniciais de importação e disseminação da COVID19 no Brasil.

Prefeitura Municipal de Natal., Secretaria Municipal de Natal., Departamento de Vigilância em Saúde. (2020). Boletim Epidemiológico 13. Disponível: https://coronavirus.natal.rn.gov.brhttps://coronavirus.nat al.rn.gov.br/docs/boletim_covid_natal_13.pdf/docs/bolet im covid natal 13.pdf.

Prefeitura Municipal de Natal., SEMURB. Secretaria Municipal de Meio Ambiente e Urbanismo. (2017). Prefeitura de Natal. Conheça Melhor Seu Bairro: Região Administrativa Sul. In: Revisão do Plano Diretor de Natal. Disponível:

https://natal.rn.gov.br/semurb/planodiretor/anexos/estu dos/CONHE $\%$ C3\%87A $\% 20$ MELHOR\%20SEU\%20BAI RRO\%20-\%20ZONA\%20SUL.pdf.

Prefeitura Municipal de Natal., SEMURB. Secretaria Municipal de Meio Ambiente e Urbanismo. (2017).
Prefeitura de Natal. Conheça Melhor Seu Bairro: Região Administrativa Oeste. In: Revisão do Plano Diretor de Natal. Disponível:

https://natal.rn.gov.br/semurb/planodiretor/anexos/estu dos/CONHE $\%$ C3\%87A\%20MELHOR\%20SEU\%20BAI RRO\%20-\%20ZONA\%200ESTE.pdf.

Prefeitura Municipal de Natal., SEMURB. Secretaria Municipal de Meio Ambiente e Urbanismo. (2017). Prefeitura de Natal. Conheça Melhor Seu Bairro: Região Administrativa Norte. In: Revisão do Plano Diretor de Natal. Disponível:

https://natal.rn.gov.br/semurb/planodiretor/anexos/estu dos/CONHE\%C3\%87A\%20MELHOR\%20SEU\%20BAI RRO\%20-\%20ZONA\%20NORTE.pdf.

Prefeitura Municipal de Natal., SEMURB. Secretaria Municipal de Meio Ambiente e Urbanismo. (2017). Prefeitura de Natal. Conheça Melhor Seu Bairro: Região Administrativa Leste. In: Revisão do Plano Diretor de Natal. Disponível:

https://natal.rn.gov.br/semurb/planodiretor/anexos/estu dos/CONHE\%C3\%87A\%20MELHOR\%20SEU\%20BAl RRO\%20-\%20ZONA\%20LESTE.pdf.

SESAP. Secretaria de Estado da Saúde Pública. RegulaRN - Leitos Regulados. (2020). Disponível: https://portalcovid19.saude.rn.gov.br/medidas/regularnsituacao-dos-leitos-do-rn/.

World Health Organization. (2020). Coronavirus disease 2019 (COVID-19) Situation Report - 1. Disponivel: https://www.who.int/docs/defaultsource/coronaviruse/situation-reports/20200121-sitrep1-2019-ncov.pdf?sfvrsn=20a99c10_4.

World Health Organization. (2020). Coronavirus disease 2019 (COVID-19) Situation Report - 510. Disponível:

https://apps.who.int/iris/bitstream/handle/10665/331685 InCoVsitrep01Apr2020-eng.pdf. 